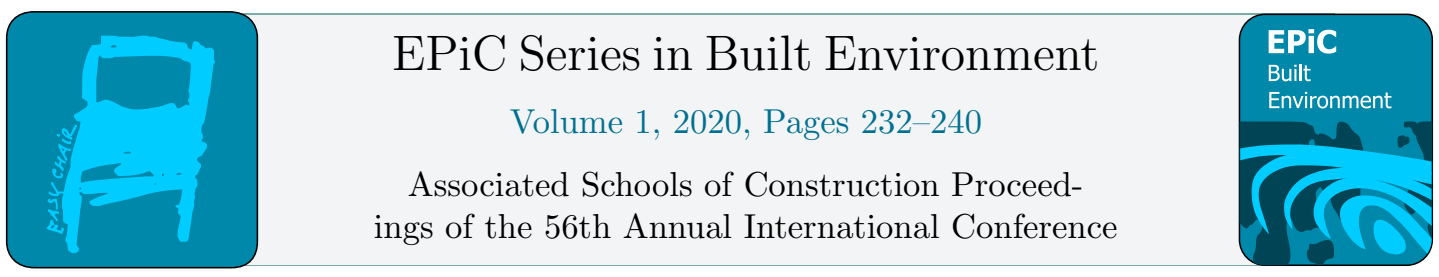

\title{
A Conceptual Framework for Transportation Infrastructure Resilience Optimization
}

\author{
Shantanu Kumar, MS and Mohammed S. Hashem M. Mehany, PhD., PMP \\ Department of Construction Management, Colorado State University, \\ Fort Collins, Colorado
}

\begin{abstract}
Limited financial resources and increased demand for transportation infrastructure maintenance and rehabilitation have complicated investment decision making in recent decades. Additionally, the cascading effects of disasters on critical infrastructure combined with insufficient funding for rehabilitation projects have intensified the situation. Meanwhile, infrastructure resiliency has emerged as a major solution to this problem and research efforts are currently implementing resilience concepts in current and future transportation infrastructure projects. Individual research studies have created models to assess investment decisions related to recovery and other facets of resilience (e.g., adaptability and robustness). However, most of these efforts have been fragmented and none have been applied on a standardized basis or been applicable to fit a standard system for infrastructure resiliency measures over different infrastructure projects (e.g. transportation) around the United States. This quantitative research builds on the Envision standardized rating system's resilience section to explore the possibilities of investment decisions influenced by adopting different resilience strategies. The novel optimization model uses mathematical modeling to assess various combinations of resilience strategies under budget constraints to find an optimal solution. The model has been successful in providing results based on user-defined priorities for cost and resilience.
\end{abstract}

Key Words: Resilience, Optimization, Transportation, Investment

\section{Introduction}

The total value of US transportation infrastructure has been estimated at approximately $\$ 7.7$ trillion in 2016 and accounts for nearly 9\% of the US Gross Domestic Product (GDP) (BTS, 2018). Aging infrastructure has increased the rehabilitation backlog to billions of dollars in recent years (ASCE, 2017). This problem has been greatly magnified by the increased frequency of disaster occurrence and its massive recovery costs (Kunreuther \& Michel-Kerjan, 2011). Consequently, infrastructure resilience emerged as the most viable solution for effective asset management due to crumbling infrastructure (Boin \& McConnell, 2007) and finance shortages, to minimize disruptions and reduce recovery costs 
(Gay \& Sinha, 2013). Infrastructure resiliency can be increased by improving an infrastructure's adaptability, robustness, recovery, and resourcefulness (Mehany, 2016) through the implementation of various strategies. Goodman (2012) indicated the value of investments in resilient structures compared to the cost of recovery and stressed the need to improve the resilience strategies when rebuilding after a disaster. Various infrastructure rating systems were needed to promote resilience thinking and minimizing losses from potential hazards (100resilientcities, 2018). Envision rating system (ISI, 2018) has been the most popular infrastructure rating system across the United States. Building a more resilient infrastructure and achieving a higher resilience or sustainability rating demands an upfront investment and financing. Berkeley et al. (2010), in the National Infrastructure Advisory Council report, identified a need for cost-effective resilience options to reduce vulnerability and increase resourcefulness. These investments pose a tradeoff in an informed selection of strategies.

These resilient strategies and their financing create a tradeoff between achieving a greater resilience and wise investment decisions which have been promoted by government incentives (Tonn et al., 2018). It has been argued that increasing resilience drives higher intital investment (Todini, 2000). In-spite of singular efforts to optimize resilience strategies, the resilience strategies have not been assessed over a standardized framework. The objective of this research is to optimize resilience for transportation infrastructure (e.g., roads, bridges and transit) under budget constraints, with the least cost possible. The Envision rating system has been used as the standardized framework to assess cost options with corresponding resilience levels and select an optimized combination of strategies under a user-defined budget.

\section{Literature Review}

\section{Resilience in Transportation}

Resilience has been defined as "the capacity of a system to absorb disturbance and reorganize while undergoing change so as to still retain essentially the same function, structure, identity, and feedbacks" (Kinzig et al., 2006; Walker et al., 2004). FHWA (2017b) defines resilience as "the ability to anticipate, prepare for, and adapt to changing conditions and withstand, respond to, and recover rapidly from disruptions".

The earliest studies on developing resilient frameworks used multi-criteria decision models for assessment of risk-mitigation (Karaca, 2005) options, which incited a need for cost-based assessment in future studies. Proag and Proag (2014) performed a cost-benefit analysis to estimate the economic efficiency of comparable projects and selecting the best option that offers the highest resilience value. Mehany (2016), suggested a unique framework that includes resiliency into the infrastructure rating systems in a more comprehensive approach which promotes robustness, resourcefulness, recovery, and adaptability in design and construction. The Infrastructure Sustainability Council of Australia (ISCA) developed an infrastructure sustainability rating tool for evaluating infrastructure sustainability and resilience through planning, design, construction, and operational phases (ISCA, 2016). The Civil Engineering Environment Quality Assessment and Award Scheme (CEEQUAL) is a rating system for European infrastructure projects developed by the Institution of Civil Engineers (ICE) and the UK Government (Mehany, 2016). The Institute for Sustainable Infrastructure (ISI) and Zofnass program for sustainable infrastructure at Harvard University developed the Envision rating system for US infrastructure systems, wherein a project's sustainability and resilience can be assessed (ISI, 2018). 
Envision is a framework that includes 64 sustainability and resilience indicators (referred to as credits) that addresses broader community issues such as human wellbeing, mobility, community development, collaboration, economy, energy, water, conservation, ecology, emissions, and resilience. This is the only system in the United States that could be applied to a plethora of infrastructure, having a total of 37 categories (e.g., roads, transit, solar, stormwater, waste recycling, parks and telecom among many others) (Mehany, 2016; ISI, 2018). This research focuses on the resilience category of Envision.

\section{Envision-Resilience Category}

The resilience category of Envision (version 3) aims at assessing both short and long-term risks associated and promotes the building of robust structures that require low maintenance (ISI, 2018). Each of the resilience credits is awarded a unique "Level of Achievement" (LOA) (among improved, enhanced, superior, conserving and restorative) based on the project performance improvement. The credit sections under the resilience category are: avoid unsuitable development (CR 2.1), assess climate change vulnerability (CR 2.2), evaluate risk and resilience (CR 2.3), establish resilience goals and strategies (CR 2.4), maximize resilience (CR 2.5) and improve infrastructure integration (CR 2.6).

The CR 2.1 credit aims to ensure the best site selection while avoiding various location-oriented threats such as permafrost, steep slope, flood-prone, and landslide-prone areas. It also assesses the effect of project development on the surrounding areas' risk triple bottom line (social, economic and environmental). The CR 2.2 credit helps the project team create a climate change vulnerability assessment plan to ensure wise economic investments for a project. The CR 2.3 credit helps the project team conduct a comprehensive, multi-hazard risk and resilience evaluation based on a common hazards list and risk evaluation steps. The LOAs are awarded based on the nature (and level) of risk and resilience assessment conducted by the project team. The CR 2.4 credit helps establish resilience goals and strategies for both at project and community level thereby decreasing losses associated with assets, health, life, and money. It also aims to transform qualitative goals into achievable quantified objectives. The CR 2.5 credit intends to implement strategies to increase system performance and robustness. Lastly, the CR 2.6 credit improves "operational relationships and strengthens the functional integration of the project". It promotes the project team to improve the systems thinking wherein the resilience goals are developed for an integrated infrastructure (and not just for a single project) to reduce the impact of cascading failures due to interdependencies (ISI, 2018). Every one of these credits (CR 2.1 through CR 2.6) has unique requirements for the five LOAs, based on which a specific number of points are awarded as shown in Table 1.

\begin{tabular}{|l|c|c|c|c|c|}
\hline \multicolumn{1}{|c|}{ Table 1: Maximum Achievable Points for each LOA } \\
\hline \multicolumn{1}{|c|}{ Resilience Credits } & Improved & Enhanced & Superior & Conserving & Restorative \\
\hline $\begin{array}{l}\text { CR 2.1 Avoid } \\
\text { Unsuitable } \\
\text { Development }\end{array}$ & 3 & 6 & 8 & 12 & 16 \\
\hline $\begin{array}{l}\text { CR 2.2 Assess Climate } \\
\text { Change Vulnerability }\end{array}$ & 8 & 14 & 18 & 20 & N/A \\
\hline $\begin{array}{l}\text { CR 2.3 Evaluate Risk } \\
\text { and Resilience }\end{array}$ & 11 & 18 & 24 & 26 & N/A \\
\hline $\begin{array}{l}\text { CR 2.4 Establish } \\
\text { Resilience Goals and } \\
\text { Strategies }\end{array}$ & N/A & 8 & 14 & 20 & N/A \\
\hline
\end{tabular}




\begin{tabular}{|l|c|c|c|c|c|}
$\begin{array}{l}\text { CR 2.5 Maximize } \\
\text { Resilience }\end{array}$ & 11 & 15 & 20 & 26 & N/A \\
\hline $\begin{array}{l}\text { CR 2.6 Improve } \\
\text { Infrastructure } \\
\text { Integration }\end{array}$ & 2 & 5 & 9 & 13 & 18 \\
\hline
\end{tabular}

Table 1 demonstrates the maximum achievable points for each resilience credit in all five LOAs. These points accurately represent the level of resilience (for each credit) of any infrastructure project, with the higher numbers representing better resilience. Various resilience strategies can be implemented with different corresponding costs to achieve these credits, all of which are subject to budget and resource constraints. Therefore, several research efforts were undertaken to optimize resilience strategies in order to build the most resilient infrastructure under the constraint of limited resources and budgets.

\section{Optimizing Resilience Strategies}

Todini (2000) showed a direct correlation between investment and resilience levels wherein increments to investment can increase resilience, but not proportionally. FHWA (2017a), in an attempt to effectively manage infrastructure assets, validated the presence of a tradeoff between investment types (e.g., preventive maintenance vs rehabilitation) and resiliency. Vugrin and Camphouse (2010) emphasized cost usage for assessing various resilience approaches to identify and define different levels of resilience. Pant (2012) analyzed resource-constrained investment options to achieve maximum resilience. Proag and Proag (2014) developed a benefit-cost analysis framework to assess the benefits of increased resilience in an uncertainty driven economics. Afrin and Yodo (2019) used a multiobjective function to maximize the resilience of water distribution networks against localized attacks and minimize recovery duration and cost. Gong and You (2018) developed a robust model to optimize the resilience and economic strategies simultaneously for a chemical process network. All of the aforementioned resilience optimization studies show the need for resilience strategy assessment and optimizing selections based on recovery costs and duration. However, the cost of different strategies, based on a standardized resilience framework such as Envision, has not been addressed. A standard system such as Envision provides a structure for assessing multiple infrastructure systems on a single platform as it includes a plethora of sustainability and resilience quantitative indicators. In an effort to maximize the resilience, this research aims to optimize the selection of various combinations of strategies (each with an associated cost) to achieve the most feasible resilience (as per the resilience section of Envision) for transportation infrastructure projects constrained by specific budgets and funding.

\section{Methodology}

As previously mentioned, this research study focusses on the tradeoff between the transportation infrastructure resilience represented by the resilience points in the Envision rating system and the cost of resiliency strategies that can be implemented to achieve such levels of resilience. Therefore, in this research study, resilience is quantified by the six credit sections under Envision's "Resilience Category". Figure 1 shows a breakdown of how each Envision resilience credit can be achieved by different combinations of resilience solutions, each contains a different set of resilience strategies that can be implemented, and each may have different associated costs. In Figure 1, every credit section (CR 2.1 to 2.6) has four resiliency combinations (making a total of $4^{6}$ i.e. 4096 total combinations), from which a single combination can be selected to achieve a certain LOA for that credit. Each combination is comprised of a set of strategies that can be implemented to improve the transportation infrastructure resilience. For example, consider achieving CR 2.3-Evaluate Risk and Resilience for a river crossing project. One of the combinations comprises improving cross-river mobility (strategy 1), improving the 
natural flow of a river (strategy 2), reducing traffic congestion (strategy 3) and integration of the project into existing roads or highways (strategy 4).

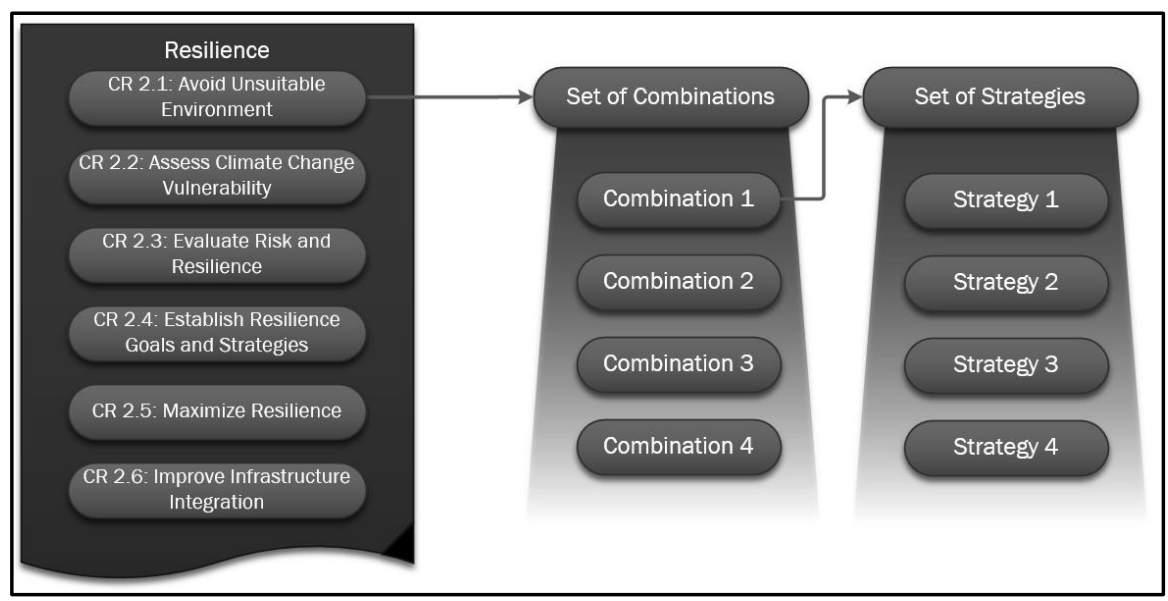

Figure 1: Resilience Breakdown and Related Measures

Similarly, every combination is created using a list of similar strategies chosen from a pool of choices (containing all relevant strategies), each having a unique total cost. A set of similar combinations (each having a different cost) that includes different sets of strategies, provides different LOA choices, resulting in a tradeoff between the investment and LOA for CR 2.3 credit. The same process is applied to all other resilience credits (CR 2.1 to CR 2.6) where each combination (comprised of a set of strategies) has a unique cost and provides a unique LOA. Hence, this research employs a bi-objective (two objectives) optimization model (Hirpa et al., 2016) to make an informed cost-based choice (among the set of combinations) that selects one combination for each of the resilience credits with an objective of maximizing the total resilience points (objective 1) with the lowest costs possible (objective 2) under a constrained budget. The user-defined Relative Importance Factors (RIFs) for resilience points and the associated cost helps in prioritizing tradeoffs to provide relevant optimized solutions. This implies that the optimized solution would change if the user provides a higher priority for resilience points or cost or both. The following section describes the mathematical formulation of the aforementioned tradeoff among the different combinations and strategies to achieve those resilience levels within the different budget constraints.

\section{Mathematical Formulation}

The bi-objective problem is formulated as mathematical functions Minimize Cost Function

$T C=\sum_{i=1}^{i=6} \cdot n_{C_{1}}\left(\operatorname{Cost}_{i}\right)$

In Equation $1 \mathrm{TC}$ represents the total cost associated with selected combination; i represents the credits (from CR 2.1 to CR 2.6 as shown in Figure 1); n represents the number of combination for each resilience credit (4 in this case); and, Cost $_{\mathrm{i}}$ is the cost associated with each combination. One combination is selected from each credit and the costs are added to get the TC. 


\section{Maximize Points Function}

$T P=\sum_{i=1}^{i=6} \cdot n_{C_{1}}\left(\right.$ Points $\left._{i}\right)$

In Equation 2, TP represents the total points associated with the selected set of combinations; i represents the credits; $n$ represents the number of combinations for each resilience credit ( 4 in this case); and Points $s_{i}$ are the points (based on a unique LOA) associated with each combination. One combination is selected from each credit and the points are added to get the TP.

\section{Maximize Objective Function}

Each of the resilience credits has a unique LOA (improved, enhanced, superior, conserving and restorative). An objective function that combines both objectives (maximizing points and minimizing cost) has been described below.

OF $=-\left(\right.$ Normalized Cost $\left.\times\left(\frac{W c}{W c+W p}\right)\right)+\left(\right.$ Normalized Points $\left.\times\left(\frac{W p}{W c+W p}\right)\right)$

Subject to:

$T C \leq$ Budget

In Equation 3, OF represents the objective function; Wc is the Relative Importance Factor (RIF) for cost and Wp is the RIF for resilience points, which are user-defined priorities for maximizing points or minimizing cost. Both cost and points are normalized with respect to their maximums to overcome a variation in data range and transform the dataset into a more coherent and parochial range ( 0 to 1 in this case). The objective function is maximized subject to a cost constraint of resilience budget (set by the user) to ensure the total cost of optimal solution does not exceed the upper limit. The option with the highest fitness value is the optimal solution. This is based on the fact that if positive numbers are arranged in descending order, the negation of the order makes them ascending. Hence if the maximum fitness value is taken into consideration, the negative sign in the fitness function achieves minimizing the cost, and the positive sign achieves maximizing the resilience credits. However, such a complex model is solved using mathematical modeling on a Python platform (discussed in the next section). The mathematical modeling of the bi-objective criteria is done using a Python 3.0 computer programmed code. Coding the model starts with an import of an excel based dataset on which the mathematical operations are performed. One of the major reasons for selecting Python as the primary programming language in this research study is the user-friendly syntax. Python syntax includes Jupyter notebook as the editor for coding and running the model, Numpy package to create the multi-objective model, and Panda package to import the data set from excel (Kumar, 2018). An exhaustive search has been used as part of mathematical modeling to find optimal solutions based on various user-defined priorities as discussed in the next section.

\section{Data Analysis}

The test dataset in Table 2 containing dummy values (showing an excerpt from the entire dataset) defines all resilience credits with associated combinations. Execution of each combination entails a specific cost and awards a unique LOA (in points). The user-defined resilience budget is set to $\$ 110,000$. The baseline for this study is defined as the budgeted cost $(\$ 110,000)$ and the maximum achievable resilience points are 74 points for this test project. Three scenarios are considered to analyze the above dataset over different user-defined priorities to find the optimal solution. 


\begin{tabular}{|c|c|c|c|c|}
\hline \multicolumn{4}{|l|}{ Table 2: Resilience Dataset } & \multicolumn{3}{|l|}{} \\
\hline Number & Credit & Combinations & Cost & LOA (points) \\
\hline \multirow{4}{*}{ CR 2.1 } & \multirow{3}{*}{$\begin{array}{c}\text { Avoid unsuitable } \\
\text { development }\end{array}$} & Combo 1 & $\$ 12,000.00$ & Restorative (16) \\
\cline { 3 - 5 } & & Combo 2 & $\$ 10,000.00$ & Superior (8) \\
\cline { 3 - 5 } & & Combo 3 & $\$ 6,500.00$ & Improved (3) \\
\cline { 3 - 5 } CR 2.2 & $\begin{array}{c}\text { Assess climate } \\
\text { change }\end{array}$ & Combo 4 & $\$ 8,000.00$ & Conserving (12) \\
\cline { 3 - 5 } & vulnerability & Combo 2 & $\$ 15,000.00$ & Enhanced (14) \\
\cline { 3 - 5 } & & Combo 3 & $\$ 18,000.000 .00$ & Superior (18) \\
\cline { 3 - 5 } & Combo 4 & $\$ 13,000.00$ & Improved (8) \\
\hline
\end{tabular}

The first scenario defines cost minimization as the top priority with RIFs being 9 and 1 for cost and resilience points respectively. The optimized result for this scenario has been shown in Table 3 . The result implies that combination 3 should be used for CR 2.1, combination 4 for CR 2.2, combination 2 for CR 2.3, combination 4 for CR 2.4, combination 4 for CR 2.5 and combination 1 for CR 2.6. The total cost obtained for this scenario is $\$ 102,500$ (the minimum achievable cost in the dataset) and the total points obtained are 53. It can be inferred that the minimum cost is achieved when the cost is defined as the highest priority.

\begin{tabular}{|c|c|c|}
\hline Scenario & Credits & $\begin{array}{c}\text { Optimized } \\
\text { Combination }\end{array}$ \\
\hline \multirow{6}{*}{$\begin{array}{c}\text { Scenario 1: } \\
\text { Cost } \\
\text { Minimization } \\
\text { (Cost:9, } \\
\text { Points:1) }\end{array}$} & CR 2.1 Avoid unsuitable development & Combo 3 \\
\hline & CR 2.2 Assess climate change vulnerability & Combo 4 \\
\hline & CR 2.3 Evaluate risk and resilience & Combo 2 \\
\hline & CR 2.4 Establish resilience goals and strategies & Combo 4 \\
\hline & CR 2.5 Maximize resilience & Combo 4 \\
\hline & CR 2.6 Improve infrastructure integration & Combo 1 \\
\hline \multirow{6}{*}{$\begin{array}{l}\text { Scenario 2: } \\
\text { Cost } \\
\text { Minimization } \\
\text { (Cost:1, } \\
\text { Points:9) }\end{array}$} & CR 2.1 Avoid unsuitable development & Combo 1 \\
\hline & CR 2.2 Assess climate change vulnerability & Combo 1 \\
\hline & CR 2.3 Evaluate risk and resilience & Combo 1 \\
\hline & CR 2.4 Establish resilience goals and strategies & Combo 4 \\
\hline & CR 2.5 Maximize resilience & Combo 1 \\
\hline & CR 2.6 Improve infrastructure integration & Combo 1 \\
\hline \multirow{6}{*}{$\begin{array}{l}\text { Scenario 3: } \\
\text { Cost } \\
\text { Minimization } \\
\text { (Cost:9, } \\
\text { Points:9) }\end{array}$} & CR 2.1 Avoid unsuitable development & Combo 4 \\
\hline & CR 2.2 Assess climate change vulnerability & Combo 4 \\
\hline & CR 2.3 Evaluate risk and resilience & Combo 2 \\
\hline & CR 2.4 Establish resilience goals and strategies & Combo 1 \\
\hline & CR 2.5 Maximize resilience & Combo 4 \\
\hline & CR 2.6 Improve infrastructure integration & Combo 1 \\
\hline
\end{tabular}

For the second scenario, the RIFs are modified to ensure resilience points have the highest priority. RIFs for cost and points being 1 and 9 respectively. The optimized solution for this scenario has been shown in Table 3. The result implies that combination 1 should be selected for CR 2.1, combination 1 for CR 2.2 and so on. The total cost obtained for this scenario is $\$ 108,000$ and the total points obtained are 74 (the maximum achievable points in the dataset). The third scenario has the same priority for cost and points with RIFs being 9 and 9 for cost and points respectively. The optimized result for this scenario has been shown in Table 3 . The result implies that combination 4 should be selected for CR 
2.1, combination 4 for CR 2.2 and so on. The total cost obtained for this scenario is $\$ 108,000$ and the total resilience points are 74 . It can be inferred that competing priorities result in maximizing the points and minimizing the cost while ensuring that the total cost is under budget.

The results show that the cost and resilience points are minimized and maximized simultaneously and a different optimal solution is obtained according to the user-defined priorities (RIFs). The dynamic nature of the python code used in this research helps achieve the minimum cost possible under the userdefined budget for every scenario.

\section{Conclusion}

The results show that the optimization model is successful in minimizing cost and maximizing resilience points based on user-defined priorities (RIFs). Additionally, it also includes a budget constraint to ensure that the optimal solution's (selected combination that comprises a set of strategies) total cost is within a pre-determined budget and according to the user's defined preferences. The model can be easily re-programmed to fit future Envision versions due to its dynamic nature. According to ISI (2018), Envision helps standardize internal accountability and assessment, incentivize project resilience, strengthens collaboration and monitors the commitment to resiliency objectives. The Envision framework provides a baseline for resilience assessment to analyze different projects across diverse geographical regions of the United States and should be useful for DOTs resilience practitioners, resilience analysts and other stakeholders such as contractors, permitting agencies, financing institutions, and insurance companies. The optimization model performs successfully within the confined scope of using exhaustive search which can evolve into evolutionary algorithms with an increase in complexity. The model's functionality is limited to the current (most updated) version of Envision and hence, it would need an update with the launch of every new version. This is currently an ongoing research wherein robustness, adaptability, recovery and resourcefulness concepts in addition to the interconnectivity among different Envision credits will be used to make the optimization model more robust for complicated infrastructure systems.

\section{References}

100resilientcities. (2018). Develop metrics for rating the resilience of infrastructure. Retrieved from https://medium.com/safer-and-stronger-cities-strategies-for-advocatin/develop-metrics-forrating-the-resilience-of-infrastructure-1bb14297b27a

Afrin, T., \& Yodo, N. (2019). Resilience-Based Recovery Assessments of Networked Infrastructure Systems under Localized Attacks. Infrastructures, 4(1), 11.

ASCE. (2017). Infrastructure Report Card. Retrieved from https://www.infrastructurereportcard.org/infrastructure-super-map/

Berkeley, A., Wallace, M., \& COO, C. (2010). A framework for establishing critical infrastructure resilience goals. Final Report and Recommendations by the Council, National Infrastructure Advisory Council.

Boin, A., \& McConnell, A. (2007). Preparing for critical infrastructure breakdowns: the limits of crisis management and the need for resilience. Journal of contingencies and crisis management, 15(1), 50-59.

BTS. (2018). TET 2018 - Chapter 8 - Value of and Investment in Transportation Infrastructure and Other Assets. Retrieved from https://www.bts.gov/transportation-economic-trends/tet-2018chapter-8-infrastructure-assets

CDOT. (2018). CDOT Resilience Program. Retrieved from https://www.codot.gov/programs/planning/cdot-resilience-program 
DHS. (2015a). Critical Infrastructure Sectors. Retrieved from https://www.dhs.gov/cisa/criticalinfrastructure-sectors

DHS. (2015b). Transportation Systems Sector-Specific Plan. Retrieved from https://www.dhs.gov/sites/default/files/publications/nipp-ssp-transportation-systems-2015508.pdf

FHWA. (2017b). FHWA Order 5520. Retrieved from https://www.fhwa.dot.gov/legsregs/directives/orders/5520.cfm.

FHWA. (2019). Building Resilient Transportation Retrieved from https://www.fhwa.dot.gov/environment/sustainability/resilience/publications/brt_brochure20 19.pdf

Gay, L. F., \& Sinha, S. K. (2013). Resilience of civil infrastructure systems: literature review for improved asset management. International Journal of Critical Infrastructures, 9(4), 330-350.

Gong, J., \& You, F. (2018). A Resilience Analysis Approach for Process Design, Integration and Optimization. Chemical Engineering Transactions, 70, 1681-1686.

Goodman, A. (2012). Calculating the cost of disaster vs. the price of resilience. Retrieved from https://www.greenbiz.com/blog/2012/12/07/Calculating-cost-disaster

Hirpa, D., Hare, W., Lucet, Y., Pushak, Y., \& Tesfamariam, S. (2016). A bi-objective optimization framework for three-dimensional road alignment design. Transportation Research Part C: Emerging Technologies, 65, 61-78.

ISCA. (2016). The IS Rating System Scheme. Retrieved from https://www.isca.org.au/is_ratings

Karaca, E. (2005). Regional earthquake loss estimation: role of transportation network, sensitivity and uncertainty, and risk mitigation. Massachusetts Institute of Technology,

Kinzig, A. P., Ryan, P. A., Etienne, M., Allison, H. E., Elmqvist, T., \& Walker, B. H. (2006). Resilience and regime shifts: assessing cascading effects. Ecology and society, 11(1).

Kumar, S. (2018). Finding a Solution for the Tradeoff Between Time, Cost and Sustainability/LEED Credits for New Construction. Colorado State University,

Kunreuther, H. C., \& Michel-Kerjan, E. O. (2011). At war with the weather: managing large-scale risks in a new era of catastrophes: MIT Press.

Mehany, M. S. H. M. (2016). Resilient, Sustainable and Green Infrastructure Concepts-A Literature Review and Proposed Preliminary Framework for a Comprehensive Infrastructure Evaluation System (CIES). Paper presented at the Construction Research Congress 2016.

Meyer, M. D., Amekudzi, A., \& O'Har, J. P. (2010). Transportation asset management systems and climate change: adaptive systems management approach. Transportation research record, 2160(1), 12-20.

Pant, S. B. (2012). Transportation network resiliency: A study of self-annealing.

Proag, S.-L., \& Proag, V. (2014). The cost benefit analysis of providing resilience. Procedia Economics and Finance, 18, 361-368.

ISI. (2018). Envision V3. Retrieved from https://sustainableinfrastructure.org/envision-version-3/

Todini, E. (2000). Looped water distribution networks design using a resilience index based heuristic approach. Urban water, 2(2), 115-122.

Tonn, G. L., Czajkowski, J. R., \& Kunreuther, H. C. (2018). Improving US Transportation Infrastructure Resilience Through Insurance and Incentives: University of Pennsylvania. Wharton School. Risk Management and Decision ....

Vugrin, E. D., \& Camphouse, R. C. (2010). THE ROLE OF RECOVERY COSTS IN OPTIMIZATION OF INFRASTRUCTURE RESILIENCE. Retrieved from

Walker, B., Holling, C. S., Carpenter, S., \& Kinzig, A. (2004). Resilience, adaptability and transformability in social-ecological systems. Ecology and society, 9(2). 